\title{
ASSOCIATION OF DERMATOMYOSITIS WITH PSORIATIC ARTHRITIS - CASE REPORT
}

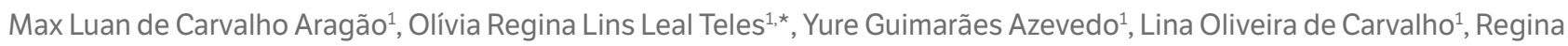
Adalva de Lucena Couto Océa ${ }^{1}$, Mônica Valéria Siqueira Santana de Vechi ${ }^{1}$, Tatiana Barbosa Castelo Branco ${ }^{1}$, José Caetano Macieira ${ }^{1}$

1.Universidade Federal de Sergipe, São Cristóvão (SE), Brazil.

*Corresponding author: oliviaa.teles@gmail.com

\section{BACKGROUND}

The association of autoimmune diseases is frequent in rheumatology, either in the form of overlapping or evolving. Isolated psoriasis can be seen in concomitance with immune-mediated rheumatologic diseases, but there are no reports in the literature of the association of inflammatory myopathy and psoriatic arthritis.

\section{METHODS}

The present report aims to discuss the case of a patient with inflammatory myopathy who, during clinical follow-up, developed psoriasis and psoriatic arthritis.

\section{RESULTS}

Patient, female, 42 years old, with dermatomyositis for about 10 years (heliotrope, upper and lower limb myopathy and calcinosis) with good evolution in use of glucocorticoid, methotrexate and chloroquine diphosphate. For two years she has been on low prednisone dose monotherapy, without using DMARDs due to the remission of the disease and the desire to become pregnant. One year ago, the patient developed polyarthritis in the hands, proximal interphalangeal (PIP)/distal interphalangeal (DIP), dactylitis in the hands and feet with deformity in the fingers, inflammatory low back pain and psoriatic lesions in the retroauricular pavilion and elbows. On physical examination, he presented enthesitis (first and seventh intercostal spaces, lateral epicondyles, femoral condyles and anterosuperior iliac crests). At hand radiography, she presented juxta-articular osteopenia with joint space reduction of PIP and DIP. Laboratory tests with negative inflammatory activity tests and negative rheumatoid factor. Magnetic resonance imaging of the pelvis with incipient bilateral sacroiliitis. Reintroduced methotrexate, we requested infectious screening (serological/tuberculin test/chest X-ray) aiming the subsequent introduction of immunobiological therapy.

\section{CONCLUSION}

This is a case of inflammatory myopathy in clinical/laboratory remission that, during follow-up, opened psoriasis/psoriatic arthritis with axial and peripheral involvement.

\section{KEYWORDS}

Dermatomyositis, Psoriatic arthritis, Association. 